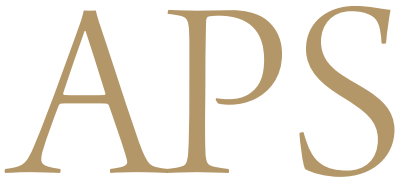

Archives of Plastic Surgery

\title{
Does Fibrin Sealant Reduce Seroma after Immediate Breast Reconstruction Utilizing a Latissimus Dorsi Myocutaneous Flap?
}

\author{
Han Gyu Cha, Sang Gue Kang, Ho Seong Shin, Moon Seok Kang, Seung Min Nam \\ Department of Plastic and Reconstructive Surgery, Soonchunhyang University College of Medicine, Seoul, Korea
}

Background The most common complication of latissimus dorsi myocutaneous flap in breast reconstruction is seroma formation in the back. Many clinical studies have shown that fibrin sealant reduces seroma formation. We investigated any statistically significant differences in postoperative drainage and seroma formation when utilizing the fibrin sealant on the site of the latissimus dorsi myocutaneous flap harvested for immediate breast reconstruction after skin-sparing partial mastectomy.

Methods A total of 46 patients underwent immediate breast reconstruction utilizing a latissimus dorsi myocutaneous island flap. Of those, 23 patients underwent the procedure without fibrin sealant and the other 23 were administered the fibrin sealant. All flaps were elevated with manual dissection by the same surgeon and were analyzed to evaluate the potential benefits of the fibrin sealant. The correlation analysis and Mann-Whitney $U$ test were used for analyzing the drainage volume according to age, weight of the breast specimen, and body mass index.

Results Although not statistically significant, the cumulative drainage fluid volume was higher in the control group until postoperative day $2(530.1 \mathrm{~mL}$ compared to $502.3 \mathrm{~mL})$, but the fibrin sealant group showed more drainage beginning on postoperative day 3 . The donor site comparisons showed the fibrin sealant group had more drainage beginning on postoperative day 3 and the drain was removed 1 day earlier in the control group.

Conclusions The use of fibrin sealant resulted in no reduction of seroma formation. Because the benefits of the fibrin sealant are not clear, the use of fibrin sealant must be fully discussed with patients before its use as a part of informed consent.

Keywords Seroma / Fibrin tissue adhesive / Mastectomy

\author{
Correspondence: Sang Gue Kang \\ Department of Plastic and \\ Reconstructive Surgery, \\ Soonchunhyang University College \\ of Medicine, 59 Daesagwan-ro, \\ Yongsan-gu, Seoul 140-743, Korea \\ Tel: +82-2-709-9283 \\ Fax: +82-2-795-3687 \\ E-mail:ksps1108@hanmail.net
}

This article was presented at the 69th congress of the Korean Society of Plastic and Reconstructive Surgeons on November 11-13, 2011 in Seoul, Korea.

No potential conflict of interest relevant to this article was reported.

Received: 25 Mar $2012 \bullet$ Revised: 21 Jun $2012 \bullet$ Accepted: 2 Jul 2012

pISSN: 2234-6163 • elSSN: 2234-6171 • http://dx.doi.org/10.5999/aps.2012.39.5.504• Arch Plast Surg 2012;39:504-508

\section{INTRODUCTION}

The latissimus dorsi myocutaneous flap remains an excellent choice for breast reconstruction despite the current popularity of the transverse rectus abdominis myocutaneous (TRAM) flap. In cases where a myocutaneous flap is necessary, the latissimus dorsi supplies the requisite well-vascularized autogenous tissue. The most common complication of this surgery is the formation of seroma in the back because of the mobility of the subscapular area with the constant movement of the shoulder girdle. This shearing effect of the overlying skin against the underlying musculature prevents the adherence of the elevated skin flaps 
to the scapula and paraspinal musculature [1]. The incidence varies from $1 \%$ [2] to $56 \%$ [3] with most authors quoting an incidence between $3 \%$ and $10 \%$ [1]. A classically accepted surgical practice for the prevention of seroma consists of inserting one or more drains during surgery [2]. However, drains create discomfort and limit mobility. Drains also may increase the possibility of infection. Therefore, a technique that allows earlier drain removal might decrease the morbidity, secondary infection, and cost, and may enhance the patient's rehabilitation and satisfaction.

Fibrin sealant has been shown to reduce seroma formation in animal models $[4,5]$. Surgeons have also evaluated the clinical effects of fibrin sealants by combining cryoprecipitated fibrinogen obtained from single-donor or autologous units and commercial bovine thrombin for a variety of indications, including seroma prevention [6].

Our unit aimed to demonstrate that the use of fibrin sealant would reduce postoperative drainage and seroma formation at the donor site in patients undergoing immediate breast reconstruction utilizing a latissimus dorsi myocutaneous flap after skin-sparing partial mastectomy. The aim was to investigate the statistically significant differences in postoperative drainage and seroma formation when utilizing the fibrin sealant on the site of the latissimus dorsi myocutaneous flap harvested for immediate breast reconstruction.

\section{METHODS}

This study was conducted at a single center with a specialized breast unit. All of the operations were performed by a single surgeon from August 2003 to February 2009. A total of 46 patients underwent immediate breast reconstruction utilizing a latissimus dorsi myocutaneous island flap. All flaps were elevated with manual dissection and electrocautery by the same surgeon and were analyzed to evaluate the potential benefits of the fibrin sealant. The patients' age, weight of the mastectomy specimen, dimensions of the latissimus dorsi flap, and body mass index (BMI) were analyzed.

All patients were female, were $\geq 30$ years old, were scheduled to undergo an elective breast procedure (skinsparing partial mastectomy with axillary node dissection), were scheduled to undergo immediate breast reconstruction utilizing a latissimus dorsi myocutaneous island flap (unilateral), did not meet any of the exclusion criteria, and had platelet counts $>100,000 / \mathrm{mL}$. The exclusion criteria were: age $<30$ years old, a history of preoperative steroid or systemic anticoagulant use a history of radiation therapy, an ongoing systemic infection at the time of surgery, being pregnant or lactating, a platelet count $\leq 100,000 / \mathrm{mL}$, or hypersensitivity to bovine protein.

Patients were divided into two groups: 23 patients who underwent surgery without fibrin sealant (control group) and 23 who were administered the aerosolized fibrin sealant (fibrin sealant group).

The same surgical technique, which involved partial mastectomy, axillary dissection, and a latissimus dorsi myocutaneous island flap procedure, was used in both groups. Three $200 \mathrm{~mL}$ Jackson-Pratt (J-P) (Barovac, Sewoon Medical, Seoul, Korea) drains were placed in the donor site, axillary dissection site, and flap coverage site (the reconstructed breast side) before closure (Fig. 1). The fibrin sealant (Tisseel, Baxter, Westlake Village, CA, US) was applied according to the manufacturer's specifications: $4 \mathrm{~mL}$ of the aerosolized fibrin sealant was sprayed (i.e., aerosolized fibrin sealant), using the manufacturer's spray set, over the donor site and the two skin flaps (Fig. 2), and then the flaps were brought together and held down with sustained pressure for a period of 5 minutes. Only three J-P drains were inserted before closure in the control group. Other procedures, such as quilting suture, which could influence the drainage volume were not performed in the two groups. A compressive dressing using elastic bandages was applied to the operation site every day. The J-P

\section{Fig. 1. The insertion of three drains}

A $200 \mathrm{~mL}$ Jackson-Pratt drain was placed in the (A) flap coverage site, (B) axillary dissection site, and (C) and latissimus dorsi muscle donor site.
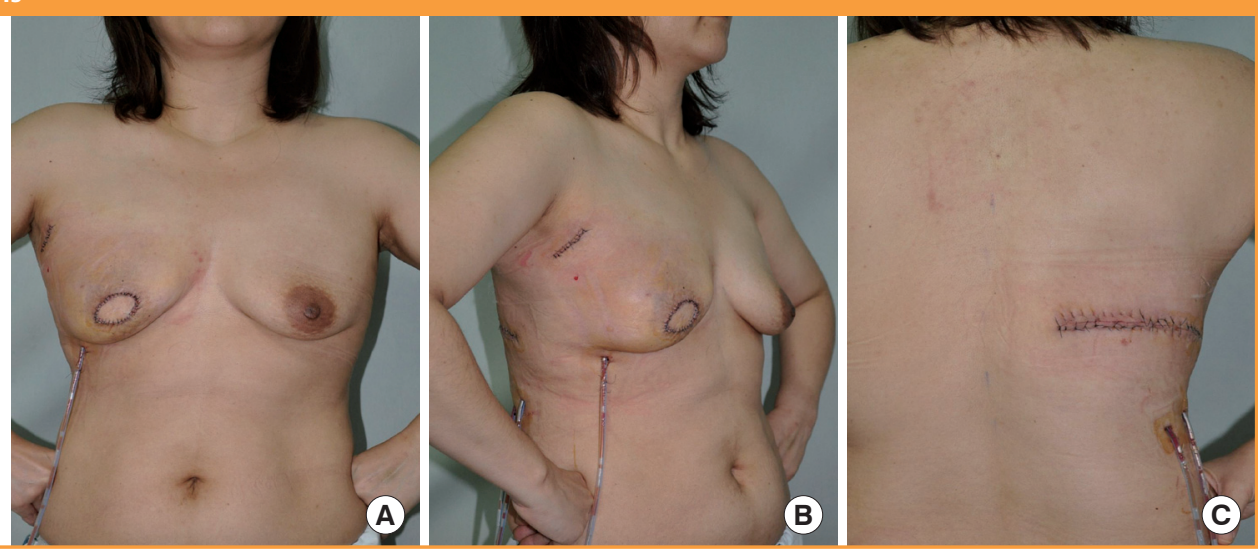
drains were removed when the drainage was less than $10 \mathrm{~mL}$ over a 24 hour period.

\section{Fibrin sealant and application}

The fibrin sealant was prepackaged with four vials in each kit: a sealer protein concentrate (human), a fibrinolysis inhibitor solution (bovine), thrombin (human), and a calcium chloride solution. Thrombin is a protease that converts fibrinogen into fibrin, and a fibrinolysis inhibitor is an enzyme that prevents fibrin from degrading prematurely.

Two components, a protein sealant and thrombin solution, were then placed in separate syringes in the dual injection system, which allowed equal amounts of the two components to be sprayed on the tissue. Mixing these two components produces viscous glue that promptly sets into a coagulum. The spray set (Duploject Preparation and Application System, Baxter) aerosolized the sealant.

\section{Statistical analysis}

The basic results are presented as mean \pm standard deviation. The normality of the data distribution was assessed using the

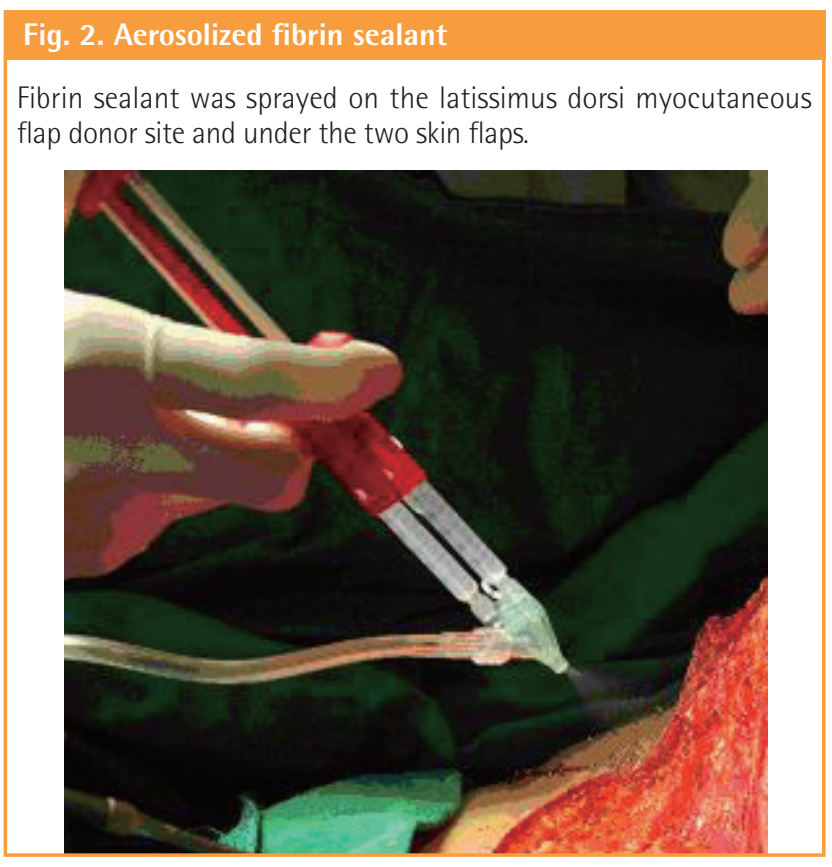

\section{Table 1. Patient demographics}

\begin{tabular}{|lcc|}
\hline Characteristic & Fibrin sealant group & Control group \\
\hline No. & 23 & 23 \\
Age & $47.5( \pm 8.2)$ & $44.5( \pm 8.8)$ \\
Body mass index & $23.8( \pm 3.1)$ & $22.8( \pm 2.6)$ \\
Weight of removed & $307( \pm 115)$ & $314( \pm 103)$ \\
breast tissue $(g)$ & & \\
\hline \multicolumn{2}{|l}{ Values are presented as number or mean \pm standard deviation. } \\
\hline
\end{tabular}

Kolmogorov-Smirnov test. The correlation analysis and MannWhitney $U$ test were used to analyze the effects of the interventions. All data were statistically analyzed using SPSS ver. 14.0 (SPSS Inc., Chicago, IL, USA), and a value of $\mathrm{P}<0.05$ was considered statistically significant.

\section{RESULTS}

Forty-six patients were enrolled in the study. All patients completed the study and were followed to the study endpoint. The patients of the two groups had similar demographics: the average age of the patients in the control group was 44.5 years (range, 33 to 65 years), and the average age in the fibrin sealant group was 47.5 years (range, 35 to 68 years) (Table 1 ). There were no statistically significant differences between the two groups in regard to age, body mass index, or the mass of the removed breast tissue. In this population set, the mean age of occurrence of breast cancer was 46 years, which corresponds to the data in the literature.

Although not statistically significant, the cumulative drainage fluid volume was higher in the control group until postoperative

\section{Fig. 3. Volume of drain output during the two week period}

The drained fluid volume was higher in the control group until postoperative day 2 , but the fibrin sealant group showed more drainage beginning on postoperative day $3(P=0.678)$.

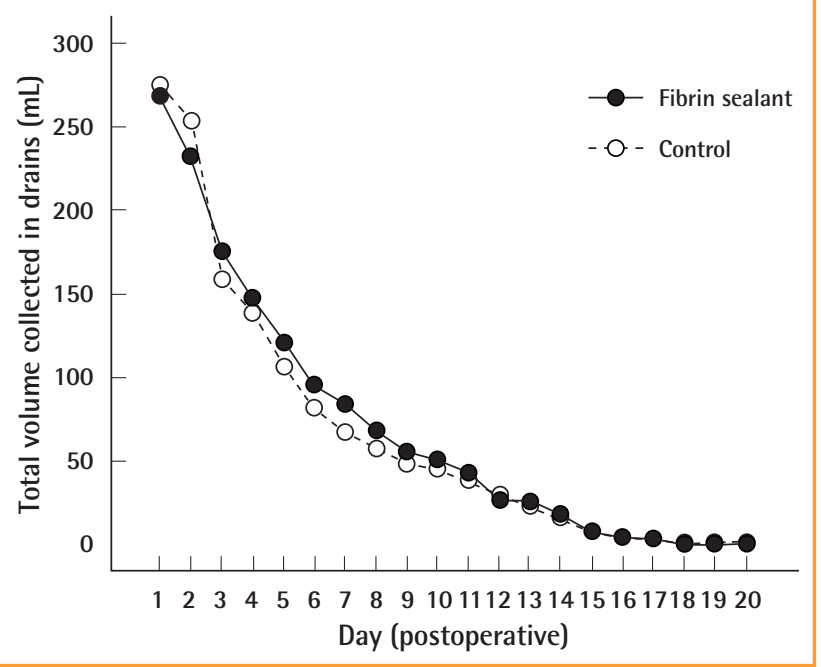

\section{Table 2. Comparison of outcome measurements}

\begin{tabular}{lcc|}
\hline Outcome & $\begin{array}{c}\text { Fibrin sealant } \\
\text { group }\end{array}$ & $\begin{array}{c}\text { Control } \\
\text { group }\end{array}$ \\
\hline $\begin{array}{l}\text { Cumulative drainage fluid volume } \\
\text { in three drains (mL) }\end{array}$ & $1,429.4( \pm 626.9)$ & $1,361.3( \pm 421.0)$ \\
Day of drain removal (day) & $12.8( \pm 3.1)$ & $11.8( \pm 3.0)$ \\
Rate of seroma formation (\%) & 0 & 0 \\
\hline Values are presented as mean \pm standard deviation. & \\
\hline
\end{tabular}




\section{Fig. 4. Volume of drain output in the donor site}

Beginning on postoperative day 3 , the drained volume was lower in the control group, and the drain was removed (when the drainage was less than $10 \mathrm{~mL}$ over a 24 hour period) 1 day earlier than the fibrin sealant group $(P=0.339)$.

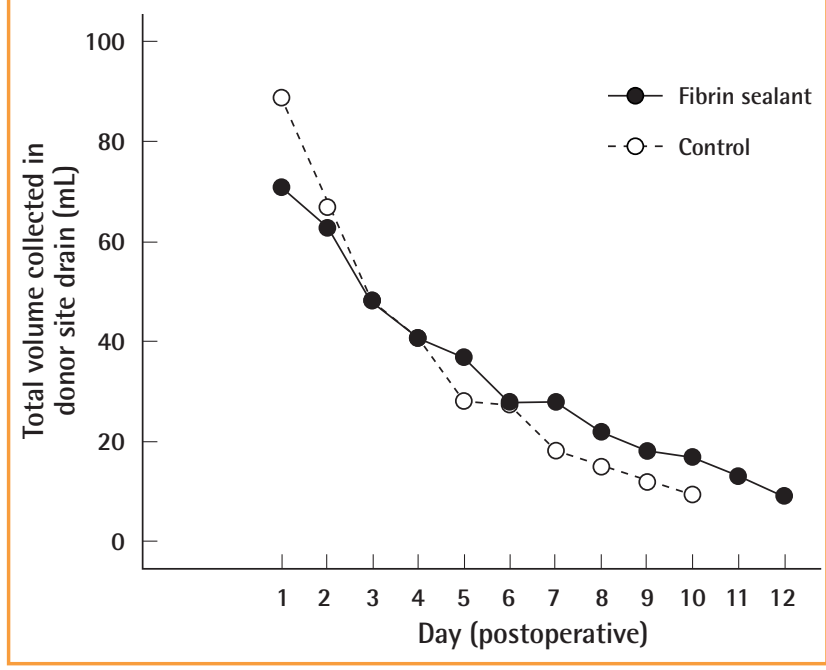

day 2 ( $530.1 \mathrm{~mL}$ compared to $502.3 \mathrm{~mL}$ ), but the fibrin sealant group showed slightly more drainage from postoperative day 3 (Fig. 3). The cumulative drainage fluid volume at the time of drain removal was $1,429 \mathrm{~mL}$ in the fibrin sealant group and $1,361 \mathrm{~mL}$ in the control group (Table 2). Comparing the donor site, where most of the fibrin sealant was applied, there was less drainage only for two days. From postoperative day 3 , the drain volume was lower in the control group, and the drain was removed an average of 1 day (11.8 compared to 12.8) earlier than in the fibrin sealant group (Fig. 4). In all cases, no delayed onset of seroma formation occurred.

Additionally, there was a correlation between the patient's $\mathrm{BMI}$ and drainage volume with a correlation coefficient of 0.36 $(\mathrm{P}<0.05)$, and between the weight of the removed breast tissue and the drainage volume with a correlation coefficient of 0.40 $(\mathrm{P}<0.05)$.

\section{DISCUSSION}

Although not life threatening, treating seroma is a significant medical expense to the patient and can result in morbidity such as pain, frequent aspiration, decreased mobility, wound dehiscence, infection, prolonged hospitalization, delayed wound healing, arm swelling, skin flap necrosis, and reoperation. The mechanism of seroma formation has been explained as a shearing effect of the cutaneous surfaces on the underlying musculature. The oozing of small vessels and the creation of a cavity caused by the removal of tissue also influences seroma formation. Seroma has been reported to be the most common complication follow- ing a latissimus dorsi flap transfer. The reported incidence varies from less than $1 \%$ [2] to up to $56 \%$ [3], and most authors quote between $3 \%$ and $10 \%$ [1].

The use of a suction drain at the latissimus dorsi donor site is routine, but drains are associated with their own complications. Not only do drains restrict activity and delay the return to normal function; they are also associated with infection, nerve injury, and flap necrosis. Seroma also frequently occurs after drain removal. Many previous studies have demonstrated that the drain fails to prevent seroma formation; the incidence of seroma requiring aspiration ranges from $15 \%$ to $83 \%$ following drain removal. Some surgeons believe these fluid collections can be treated using "benign neglect" [7], but most surgeons tend to aspirate significant seromas. Slavin [8] demonstrated that fluid collections greater than 75 to $100 \mathrm{~mL}$ may lead to wound dehiscence, implant exposure, discomfort, and infection and thus suggested draining such seromas. Intervention for seromas usually consists of needle aspiration or drainage, but this technique always carries the risk of iatrogenic complications such as pneumothorax or hemothorax.

Variousapproaches have been taken in an attempt to reduce seroma formation including external compression, arm immobilization, flap-tacking sutures, avoidance of electrocautery, use of fibrin sealant, talc poudrage [9], and the quilting technique [10]. Recently, minimal access harvesting of the latissimus dorsi flap has been attempted to minimize donor site seroma [11]. None of these techniques have been proven in a clinical situation or have gained popularity. The talc poudrage tended to cause complications associated with the chronic inflammatory response. Recently, a quilting technique is commonly utilized to reduce seroma formation, and studies have shown this method to be effective in a clinical setting [10]. However, this technique contributes to lengthening the operative time by approximately 20 minutes. The quilting sutures need to be placed very accurately, especially when large skin paddles are harvested; otherwise, donor-site closure cannot be achieved.

Fibrin sealant, a two-component tissue adhesive composed of fibrinogen and thrombin, has been used in a number of surgical settings primarily to achieve hemostasis and to seal tissues. The process by which fibrinogen and thrombin combine in the presence of factor XIII and calcium chloride to form fibrin sealant has been described extensively in the literature [12]. The mode of action of the fibrin sealant is two-pronged: it reduces the dead space between two planes by means of tissue adhesion, which coapts the elevated skin flap to the underlying tissues, and it reduces hematoma and serous oozing from the involved surfaces by enhancing hemostasis and blocking the lymph channels responsible for drainage into the operative site. The fibrin 
forms a strong bond that interlocks with the molecular structure of surgically damaged tissue and seals open tissue channels. The sealant effect is dependent on the fibrinogen concentration and clotting time on the thrombin concentration.

Several human clinical studies have been performed with commercial fibrin sealants in patients undergoing breast surgery (not breast reconstruction, but other procedures such as mastectomy or axillary dissectionwith conflicting results. Some studies have reported that fibrin sealant was found to be effective in reducing either wound drainage or length of hospital stay $[13,14]$. In contrast, fibrin sealant was not found to be effective in other studies [15].

Few studies have been performed in a clinical setting to demonstrate the efficacy of fibrin sealant in reducing complications of large surgically created dead spaces similar tothe latissimus dorsi donor site. We designed this study with the fibrin sealant as the only variable, excluding any other procedure that may influence the result. The study was limited to patients 30 years or older, in whom the risk of breast cancer is higher, and is also the recommended age for a routine screening test. As mentioned above, we excluded all the systemic factors and other procedures that might influence the volume of drainage. Consequently, it is meaningful that our study represents the largest population among the studies related to the latissimus dorsi donor site and the fibrin sealant as a single variable. A possible reason for the result that fibrin sealant had no effect in reducing seroma is that the fibrin sealant itself may elicit a tissue reaction, similar to a foreign body reaction, causing more fluid secretion. Another possible explanation is that the glue started to dissolve and was evacuated by the suctions during the surgery or by the drains. Different donor site surface areas and delayed wound closure might also have contributed to the result.

The fact that the patients' BMI and weight of the removed tissue had a correlation with the drain output volume demonstrates that the large surface area of the latissimus dorsi donor site should be taken into consideration in deciding on the dose of fibrin sealant. The optimal dose per unit area should be calculated along with the exact donor site surface area.

Seroma is a frustrating complication of many surgical procedures. We have shown that in immediate breast reconstruction with the latissimus dorsi myocutaneous flap, the fibrin sealant did not significantly decrease the drain output or hospitalization stay. In conclusion, because the benefits from the fibrin sealant are not clear, the use of fibrin sealant must be fully discussed with the patients before its use, as part of informed consent.

\section{REFERENCES}

1. Scheflan M, Kalisman M. Complications of breast reconstruction. Clin Plast Surg 1984;11:343-50.

2. Davis JP, Nield DV, Garth RJ, et al. The latissimus dorsi flap in head and neck reconstructive surgery: a review of 121 procedures. Clin Otolaryngol Allied Sci 1992;17:487-90.

3. Salmi A, Tukiainen E, Harma M, et al. A prospective study of changes in muscle dimensions following free-muscle transfer measured by ultrasound and CT scanning. Plast Reconstr Surg 1996;97:1443-50.

4. Kulber DA, Bacilious N, Peters ED, et al. The use of fibrin sealant in the prevention of seromas. Plast Reconstr Surg 1997;99:842-9.

5. Wang JY, Goodman NC, Amiss LR Jr, et al. Seroma prevention in a rat mastectomy model: use of a light-activated fibrin sealant. Ann Plast Surg 1996;37:400-5.

6. Spotnitz WD. Fibrin sealant in the United States: clinical use at the University of Virginia. Thromb Haemost 1995;74:482-5.

7. Reddick LP. Seroma after latissimus dorsi myocutaneous flap for breast reconstruction. Plast Reconstr Surg 1990;85:826.

8. Slavin AS. Seroma after latissimus dorsi myocutaneous flap for breast reconstruction-reply (letter). Plast Reconstr Surg 1990;85:826.

9. Coons MS, Folliguet TA, Rodriguez C, et al. Prevention of seroma formation after dissection of musculocutaneous flaps. Am Surg 1993;59:215-8.

10. Dancey AL, Cheema M, Thomas SS. A prospective randomized trial of the efficacy of marginal quilting sutures and fibrin sealant in reducing the incidence of seromas in the extended latissimus dorsi donor site. Plast Reconstr Surg 2010; 125:1309-17.

11. Friedlander L, Sundin J. Minimally invasive harvesting of the latissimus dorsi. Plast Reconstr Surg 1994;94:881-4.

12. Radosevich M, Goubran HI, Burnouf T. Fibrin sealant: scientific rationale, production methods, properties, and current clinical use. Vox Sang 1997;72:133-43.

13. Gilly FN, Francois Y, Sayag-Beaujard AC, et al. Prevention of lymphorrhea by means of fibrin glue after axillary lymphadenectomy in breast cancer: prospective randomized trial. Eur Surg Res 1998;30:439-43.

14. Gioffre Florio MA, Mezzasalma F, Manganaro T, et al. The use of fibrin glue in the surgery of breast carcinoma. G Chir 1993;14:239-41.

15. Vaxman F, Kolbe A, Stricher F, et al. Does fibrin glue improve drainage after axillary lymph node dissection? Prospective and randomized study in humans. Eur Surg Res 1995;27:346-52. 